\title{
On the regimes of underwater explosion for a submerged slender structure by pulsating bubble
}

\author{
Bing-Chuan Nie, Jia-Chun Li ${ }^{*}$, Hui-Qin Zhang \\ Key Laboratory for Mechanics in Fluid Solid Coupling System, Institute of Mechanics, Chinese Academy of \\ Sciences, No. 15 Beisihuanxi Road, Beijing 100190, China
}

\section{A R T I C L E I N F O}

\section{Article history:}

Received 5 November 2014

Received in revised form 29 May 2015

Accepted 24 July 2015

Available online xxx

\section{Keywords:}

Submarine

Similarity analysis

Cauchy number

Scaling law

Far-field

\begin{abstract}
A B S T R A C T
Three regimes: near-, middle- and far-fields of underwater explosion are proposed in this study aiming at providing an overview on the responses of submerged slender structure by pulsating bubble. In the near-field, the material starts to yield, thus leading to structure breakdown immediately; remarkable structural global elastic deformation occurs in the middle-field as well as substantial movement; and a structure moves as a rigid body with negligible deformation for the far-field. Equivalent dimensionless parameters are obtained by two different dimensional analysis methods, among which a dominant similarity parameter is found out. Thus, a scaling law providing us with a relation between structural global response and the dominant similarity parameter is yielded, which can be used for demarcating the three regimes quantitatively. To demonstrate, three models corresponding to typical submarine parameters are performed in the case studies. Quantitative criterion of the three regimes is presented along with the regime diagrams. The structural global response features such as the deformation and maximal acceleration/speed of different regimes are provided as well.
\end{abstract}

(c) 2015 Elsevier Ltd. All rights reserved.

* Corresponding author. Tel.: +86 01082544201.

E-mail address: jcli05@imech.ac.cn (J.-C. Li). 


\section{Introduction}

Shock, pulsating bubble and subsequent jet are three well-known processes of damage in an underwater explosion. When the failures due to shock and jet are localized, a structure tends to globally respond to pulsating bubble. As we know, an explosive bubble at first expands owing to the high pressure of detonative gas inside and then contracts from an over-expanding state under high ambient hydraulic pressure to an over-contraction state. The expanding-contracting cycle repeats several times in this way. It is estimated that about two-thirds of the bubble energy are dissipated in the first period. The bubble duration is of the order of one second, much longer than that of shock or jet. Moreover, if the bubble frequency is in the neighborhood of the eigen-frequency of a slender structure or the explosion is violent (for example large TNT exploded nearby), strong or even destructive "whipping response" could be excited.

Earlier theoretical studies on bubble dynamics were concerned with the bubble's migration and oscillation by assuming that the water is incompressible and the bubble maintains spherical shape in its life [1]. Later, non-spherical effects [2] and also the roles of compressibility in both external water and internal gas were examined [3,4]. Numerical method of explosive bubble simulation is booming during the last three decades. Boundary Element Method (BEM) has been proved an effective tool to reproduce the bubble process [5-7]. Computational Fluid Dynamics (CFD) with interface-capture algorithms has been adopted in explosion bubble modeling [8]. Moreover, the meshless method seemingly promising for the simulation of a collapsing bubble has also been developed [9]. Foregoing new advancements enhance modeling capability and enrich the contents of bubble dynamics, thus further deepening the understanding of bubble's migration and deformation features especially for the bubble nearby boundaries or in the bubble's extreme collapse phase.

Bubble dynamics studies also push forward the exploration of global response of a submerged slender structure as shown in Fig. 1. The earliest one can be traced back to the work on whipping response by Hick [10]. Then, Vernon examined the fluid velocity field by a pulsating bubble under the incompressible assumption aiming at establishing the whipping response of a nearby surface ship [1]. Moreover, the velocity field for compressible fluid was obtained by the method of matched asymptotic expansions [11], but the results have not been used in global structure response researches so far. Global response of a surface ship was analyzed by estimating the bubble loading by Vernon's method and simplifying the structure as a Bernoulli-Euler beam as well as finite element method [12-14]. Also, BEM was used to estimate bubble loading in the structural global response studies $[5,6]$. All those works primarily focused on the deformation of a specific structure with less universality. They also scarcely discussed the distribution and amplitude of the acceleration and speed response, which are closely related to equipment failure and crew's injury onboard. Furthermore, structural reverse response denoting the structural displacement has an opposite direction to the flow velocity is observed experimentally, but not well discussed yet for both small fixed plates [5] and large surface warships [15].

In recent years, the applications of high strength material and blast-resistant structure remarkably enhanced the local strength of ships and submarines. So the resistant ability to global damage has become a noteworthy challenge. Instead of discussing the global response for a specific slender

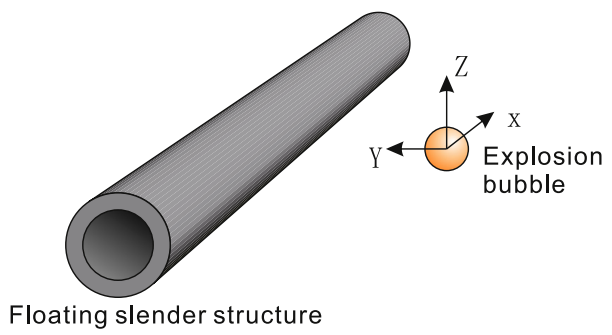

Fig. 1. A submerged floating slender structure subjected to underwater explosion bubble located at the symmetric plane. Z-axis is vertical to the free surface, and $\mathrm{X}$-axis is parallel to neutral axis of the structure. 
structure, we prefer to explore explosive regimes (near-/middle-/far-fields) of submerged slender structures in this study, aiming at providing an overview on the global response of slender structure by pulsating bubble. The results are also expected to be useful in understanding the physical mechanism and improving the blast-resistant performance of structure design.

This paper is organized as follows. In Section 2, some dimensionless numbers are obtained by both similarity analysis and governing equations derivation. The three regimes: near-, middle- and far-fields of underwater explosion corresponding to different global response features are quantitatively defined in Section 3 based on the scaling law. In Section 4, the underwater explosion regimes are used and validated in case studies of typical submarines. Furthermore, the characteristics of the middle-field and far-field are presented. Finally, the summary and conclusions in Section 5 bring this study to an end.

\section{Derivation of the dimensionless numbers}

\subsection{Similarity analysis}

Since the time scale of the shock is of order of millisecond and structural response as well as cavitation caused by shock is localized [16], the shock's influence on the subsequent response by bubble load is neglected. Thus, there are four groups of independent parameters manipulating the global response of a slender structure subjected to bubble loading. They can be listed as below:

\section{1) Bubble loading related parameters}

$u_{m}$ : the maximal flow speed at the location of the structure depending on charge mass, explosion distance and depth. Its formula will be given in Section 2.3.

$f_{b}$ : bubble pulsation frequency which can be estimated according to Eq. (18).

\section{2) Structural geometry parameters}

$L:$ the length of the slender structure,

$R$ : outer radius of the structural cross-section,

$A$ : area of the cross-section,

$I$ : moment of inertia of area.

For a given cross section shape, only two among $A, r, R, \delta$ are independent, where $r$ internal radius and $\delta$ equivalent thickness.

\section{3) Structural material parameters}

Under the assumption that the material is isotropic, only two of the three material constants are independent. Hence we use the elastic modulus and the shear modulus here.

$\rho$ : the material density,

$E$ : the elastic modulus,

$G$ : the shear modulus,

$\sigma_{Y}:$ the yield stress.

\section{4) Fluid medium parameters}

As we known, the Mach number of the bubble-induced spherical water flow is of order $O\left(10^{-1}\right)$ or less nearby the bubble [7] and the flow speed drops rapidly with distance. On the other hand, the decaying shock modeled as "quasi-acoustic" at standoff larger than 15 times of the charge radius can hardly bring significant entropy increase of water [3]. Furthermore, the surrounding water can be regarded as adiabatic due to "cooling-down" of the gas interior during expansion and the minor heat diffusion rate. Thus the incompressibility assumption is introduced for water out of bubble. Besides, as 
the structure is sufficiently large, we assume the water is inviscid. Consequently, the acoustic speed and viscous coefficient vanish.

$\rho_{w}$ : the water density.

The global responses $F_{i}$ (such as stress, acceleration, speed) can be expressed as

$$
F_{i}=f_{i}\left(u_{m}, f_{b}, L, R, A, I, \rho, E, G, \sigma_{Y}, \rho_{w}\right) .
$$

In accordance with the Mises criterion, if $\sigma_{m}$ exceeds $\sigma_{Y}$, yield begins. Where $\sigma_{m}$ reads

$$
\sigma_{m}=\max \left\{\frac{\sqrt{2}}{2} \sqrt{\left(\sigma_{1}-\sigma_{2}\right)^{2}+\left(\sigma_{2}-\sigma_{3}\right)^{2}+\left(\sigma_{1}-\sigma_{3}\right)^{2}}\right\},
$$

in which $\sigma_{1}, \sigma_{2}$ and $\sigma_{3}$ are the principal stresses. Then, Eq. (1) is rewritten as Eq. (3) with curiosity on the stresses.

$$
\sigma_{m}=f\left(u_{m}, f_{b}, L, R, A, I, \rho, E, G, \sigma_{Y}, \rho_{w}\right) .
$$

Taking $\rho_{w}, u_{m}$ and $L$ as a unit system, Eq. (3) is transformed into the dimensionless formula below

$$
\frac{\sigma_{m}}{\rho_{w} u_{m}^{2}}=f\left(\frac{f_{b}}{u_{m} / L}, \frac{R}{L}, \frac{A}{L^{2}}, \frac{I}{L^{4}}, \frac{\rho}{\rho_{w}}, \frac{E}{\rho_{w} u_{m}^{2}}, \frac{G}{\rho_{w} u_{m}^{2}}, \frac{\sigma_{Y}}{\rho_{w} u_{m}^{2}}\right) .
$$

Altering the form of Eq. (4), eventually, we have the relation

$$
\frac{\sigma_{m}}{\sigma_{Y}}=f\left(\frac{R f_{b}}{u_{m}}, \frac{R}{L}, \frac{A}{R^{2}}, \frac{I}{R^{4}}, \frac{\rho}{\rho_{w}}, \frac{E}{\rho_{w} u_{m}^{2}}, \frac{G}{\rho_{w} u_{m}^{2}}, \frac{\sigma_{Y}}{\rho_{w} u_{m}^{2}}\right),
$$

in which $R f_{b} / u_{m}$ reflects the influence of bubble frequency, and $\rho / \rho_{w}$ reflects the effect of fluid-structure interaction. $E /\left(\rho_{w} u_{m}^{2}\right)$ and $\sigma_{Y} /\left(\rho_{w} u_{m}^{2}\right)$ are the inverse of Cauchy and damage numbers, respectively, which are widely used in describing the structural elastic and plastic responses independently. In some works, being multiplied by the square of the half of the slenderness ratio, the damage number is developed to include the geometrical influence [17], which is used to predicts the dynamic plastic response of perfectly rigid-plastic beams effectively. $G /\left(\rho_{w} u_{m}^{2}\right)$ describes shear effect corresponding to "tensile" effect by $E /\left(\rho_{w} u_{m}^{2}\right)$. It is an arduous task to estimate the stress by Eq. (5), because as many as eight dimensionless numbers exist in this complicated relation.

\subsection{Governing equations of the structural response}

Without loss of generality for global response study of a slender structure, Euler-Bernoulli beam, Rayleigh beam, shear beam or Timoshenko beam can be used, which are four widely used engineering models depending on whether taking shear and rotation effects into account. For structures with slenderness ratio over 100, the Euler-Bernoulli beam can be used; while for small slenderness ratio structures, both shear beam and Timoshenko beam can be used [18]. The length-diameter ratio of a submarine is always between 6 and 7 as the requirement of minimum total resistance [19]. We here simplify a submarine as a hollow cylindrical Timoshenko beam with the shear and rotation effects discussed detailedly in Section 3.1. The equivalent thickness $\delta$ of the beam can be obtained in line with the submarine's moment of inertia of area. Consequently, the governing equations are

$$
\left\{\begin{array}{l}
\frac{\partial}{\partial x}\left[k G A\left(\frac{\partial w}{\partial x}-\theta\right)\right]+q=\rho A \frac{\partial^{2} w}{\partial t^{2}}, \\
\frac{\partial}{\partial x}\left(E I \frac{\partial \theta}{\partial x}\right)+k G A\left(\frac{\partial w}{\partial x}-\theta\right)+m=\rho I \frac{\partial^{2} \theta}{\partial t^{2}},
\end{array}\right.
$$


where $w$ is the transverse deflection, $\theta$ is the rotational angle of the cross-section, $q$ is the transverse force, $m$ is the external moment, and $k$ is the shape factor for which one may refer to reference [18].

The three dimensional spherical flow can be approximated to a two dimensional flow in each crosssection for a slender structure, further to a plane flow issue as shown in Fig. 2 when the explosion distance is large. Then, the transverse force $q$ can be written as [20].

$$
q(x, t)=\left(\rho A+m_{a}\right) \dot{u}-m_{a} \frac{\partial^{2} w}{\partial t^{2}},
$$

where $\dot{u}$ is the normal acceleration of the plane flow in each cross-section with the axial flow disregarded, $m_{a}$ is the added mass equals to $\pi \rho_{w} R^{2}$ for a hollow cylindrical shaped cross-section.

Substituting Eq. (7) and $m=0$ into Eq. (6), we have

$$
\left\{\begin{array}{l}
\frac{\partial}{\partial x}\left[k G A\left(\frac{\partial w}{\partial x}-\theta\right)\right]+\left(\rho A+m_{a}\right) \dot{u}=\left(\rho A+m_{a}\right) \frac{\partial^{2} w}{\partial t^{2}} \\
\frac{\partial}{\partial x}\left(E I \frac{\partial \theta}{\partial x}\right)+k G A\left(\frac{\partial w}{\partial x}-\theta\right)=\rho I \frac{\partial^{2} \theta}{\partial t^{2}}
\end{array}\right.
$$

Introducing the following dimensionless quantities: $W=w / R, X=x / L, T=t u_{m} / R, M_{a}=m_{a} /\left(\rho_{w} R^{2}\right)$, $\rho^{\prime}=\rho / \rho_{w}, G^{\prime}=G /\left(\rho_{w} u_{m}^{2}\right), E^{\prime}=E /\left(\rho_{w} u_{m}^{2}\right), A^{\prime}=A / R^{2}, \dot{U}=\dot{u} /\left(u_{m} f_{b}\right), I^{\prime}=I / R^{4}$, then, Eq. (8) becomes

$$
\left\{\begin{array}{l}
\frac{k G^{\prime} A^{\prime}}{\rho^{\prime} A^{\prime}+M_{a}} \frac{R}{L}\left(\frac{R}{L} \frac{\partial^{2} W}{\partial X^{2}}-\frac{\partial \theta}{\partial X}\right)+\frac{R f_{b}}{u_{m}} \dot{U}=\frac{\partial^{2} W}{\partial T^{2}} \\
\frac{E^{\prime} I^{\prime}}{\rho^{\prime} A^{\prime}+M_{a}} \frac{R^{2}}{L^{2}} \frac{\partial^{2} \theta}{\partial X^{2}}+\frac{k G^{\prime} A^{\prime}}{\rho^{\prime} A^{\prime}+M_{a}}\left(\frac{R}{L} \frac{\partial W}{\partial X}-\theta\right)=\frac{\rho^{\prime} I^{\prime}}{\rho^{\prime} A^{\prime}+M_{a}} \frac{\partial^{2} \theta}{\partial T^{2}} .
\end{array}\right.
$$

Suggesting the dimensionless numbers $E_{n}, G_{n}, I_{n}$ and $f_{n}$ as

$$
\begin{aligned}
& E_{n}=\frac{E^{\prime} I^{\prime}}{\rho^{\prime} A^{\prime}+M_{a}} \frac{R^{4}}{L^{4}}, \\
& G_{n}=\frac{k G^{\prime} A^{\prime}}{\rho^{\prime} A^{\prime}+M_{a}} \frac{R^{2}}{L^{2}},
\end{aligned}
$$

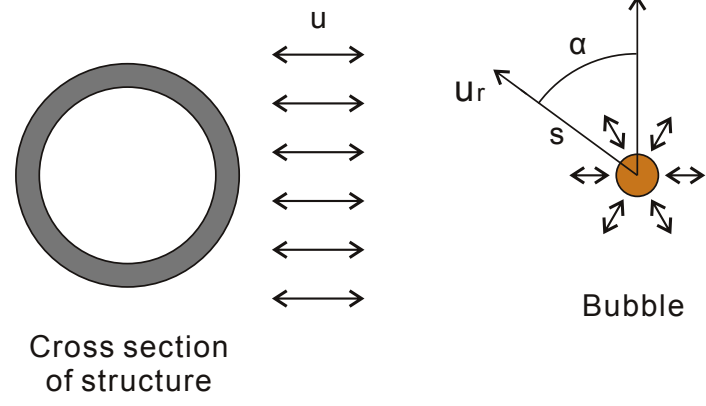

Fig. 2. Interaction between the explosion bubble flow and a cross section. The radial flow can be considered as a two dimensional plane flow in each cross-section of the beam. 


$$
\begin{aligned}
& I_{n}=\frac{\rho^{\prime} I^{\prime}}{\rho^{\prime} A^{\prime}+M_{a}} \frac{R^{2}}{L^{2}}, \\
& f_{n}=\frac{R f_{b}}{u_{m}} .
\end{aligned}
$$

Eq. (9) is reduced to

$$
\left\{\begin{array}{l}
G_{n}\left(\frac{\partial^{2} W}{\partial X^{2}}-\frac{L}{R} \frac{\partial \theta}{\partial X}\right)+f_{n} \dot{U}=\frac{\partial^{2} W}{\partial T^{2}} \\
E_{n} \frac{\partial^{2} \theta}{\partial X^{2}}+G_{n}\left(\frac{R}{L} \frac{\partial W}{\partial X}-\theta\right)=I_{n} \frac{\partial^{2} \theta}{\partial T^{2}}
\end{array}\right.
$$

Ultimately, eliminating $\theta$ in Eq. (14), we have

$$
E_{n} \frac{\partial^{4} W}{\partial X^{4}}-\left(\frac{E_{n}}{G_{n}}+I_{n}\right) \frac{\partial^{4} W}{\partial X^{2} \partial T^{2}}+\frac{\partial^{2} W}{\partial T^{2}}+\frac{I_{n}}{G_{n}} \frac{\partial^{4} W}{\partial T^{4}}=\frac{I_{n} f_{n}}{G_{n}} \frac{\partial^{2} \dot{U}}{\partial T^{2}}-\frac{E_{n} f_{n}}{G_{n}} \frac{\partial^{2} \dot{U}}{\partial X^{2}}+f_{n} \dot{U} .
$$

Similarity parameters $E_{n}, G_{n}, I_{n}$ and $f_{n}$ in Eq. (15) are the governing factors of structural response. And this group of dimensionless numbers are actually equivalent to the dimensionless numbers shown in Eq. (5) which are derived by similarity analysis if the damage number is discarded. Damage number vanishes in Eq. (15), because the Timoshenko beam model is an elastic beam model. Therefore, we conclude that the dimensionless stress in the elastic stage is governed by

$$
\frac{\sigma_{m}}{\sigma_{Y}}=f\left(f_{n}, E_{n}, G_{n}, I_{n}\right)
$$

The dimensionless numbers at the right side can be readily found out if the maximal flow speed $u_{m}$ is estimated as in Section 2.3.

\subsection{Flow velocity induced by a pulsating bubble}

At a submarine's operating depth of $300 \mathrm{~m}$ or so below the sea surface, the effect of free surface can be neglected. Based on the assumptions that the bubble maintains spherical shape with adiabatic gas in the interior, the ambient water is incompressible and inviscid, we examined the flow speed induced in the first bubble period, during which majority of the bubble energy dissipates.

The maximal bubble radius $a_{\max }$ in meter and pulsating period $T_{b}$ in second can be estimated by the following equations, respectively [21].

$$
\begin{aligned}
& a_{\max }=K_{1} \frac{C^{1 / 3}}{(H+10.3)^{1 / 3}}, \\
& T_{b}=K_{2} \frac{C^{1 / 3}}{(H+10.3)^{5 / 6}} .
\end{aligned}
$$

where $H$ the explosion depth in meter, $C$ the charge weight in kilogram, $K_{1}$ and $K_{2}$ are constants dependent on the charge properties. For TNT with density of $1.57 \mathrm{~g} / \mathrm{cm}^{3}, K_{1}$ and $K_{2}$ equal to $3.36 \mathrm{~m}^{4 / 3}$ / $\mathrm{kg}^{1 / 3}$ and $2.11 \mathrm{~m}^{5 / 6} \mathrm{~s} / \mathrm{kg}^{1 / 3}$, respectively. 
Since the volume of the bubble $V(t)$ can be reasonably approximated by a half sine function as [22]

$$
V(t)=V_{0}+\left(V_{\max }-V_{0}\right) \sin \left(\frac{\pi}{T_{b}} t\right),
$$

where $V_{0}$ and $V_{\max }$ are the initial charge and maximal bubble volumes. The bubble radius can be derived from

$$
a(t)=\left[\frac{3 V_{0}}{4 \pi}+\left(a_{\max }^{3}-\frac{3 V_{0}}{4 \pi}\right) \sin \left(\frac{\pi}{T_{b}} t\right)\right]^{1 / 3} .
$$

The Lagrange's function of the bubble dynamics reads

$$
L a=E_{k}-E_{p},
$$

where $E_{k}$ and $E_{p}$ are the kinetic and potential energies, respectively, given as [1].

$$
\begin{aligned}
& E_{k}=2 \pi \rho_{w} a^{3} \dot{a}^{2}+\frac{\pi}{3} a^{3} \dot{z}^{2}, \\
& E_{p}=\frac{4}{3} \pi \rho_{w} a^{3} g z+\frac{\kappa}{\gamma-1} \frac{C^{\gamma} 3^{\gamma-1}}{(4 \pi)^{\gamma-1} a^{3(\gamma-1)}} .
\end{aligned}
$$

In which $g$ is the gravity acceleration, $\gamma$ is the ratio of specific heats, $\kappa$ is a constant depending on the explosive material, and $\dot{z}$ is the vertical migration speed.

Then, the migration equation of the bubble can be written as

$$
\frac{d}{d t}\left(\frac{\partial L a}{\partial \dot{z}}\right)-\frac{\partial L a}{\partial z}=Q_{z}
$$

where $Q_{z}$ is the drag force of a bubble during its upward movement. Usually, the migration speed is so small that the drag force can be neglected in the first period [3].

Finally, we have the migration speed $\dot{z}$ expressed as

$$
\dot{z}=\frac{2 g}{a^{3}}\left[\frac{3 V_{0}}{4 \pi}-\frac{T_{b}}{\pi}\left(a_{m}^{3}-\frac{3 V_{0}}{4 \pi}\right) \cos \left(\frac{\pi t}{T_{b}}\right)+\frac{T_{b}}{\pi}\left(a_{m}^{3}-\frac{3 V_{0}}{4 \pi}\right)\right] .
$$

According to Eqs. (20) and (25), the variation of a bubble radius and its migrating displacement are illustrated in Fig. 3 for $226.7 \mathrm{~kg}$ TNT detonated at $45.7 \mathrm{~m}$ under the sea surface, And both agree well with the results from Hunter and Geers [11].

Considering the bubble as a pulsating and migrating source, the velocity potential $\phi$ of the ambient fluid flow can be expressed as

$$
\phi=-\frac{a^{2} \dot{a}}{S}-\frac{a^{3} \dot{z}}{2 S^{2}} \cos \alpha,
$$

where $S$ is the distance from the position of interest to the bubble center, and $\alpha$ is the angle between radial direction and the $z$-axis as shown in Fig. 2. Then, we have the radial flow speed of surrounding water

$$
u_{r}=\frac{1}{3 S^{2}} \frac{\pi}{T_{b}}\left(a_{m}^{3}-\frac{3 V_{0}}{4 \pi}\right) \cos \left(\frac{\pi t}{T_{b}}\right)+\frac{2 g \cos \alpha}{S^{3}}\left[\frac{3 V_{0}}{4 \pi} t-\frac{T_{b}}{\pi}\left(a_{m}^{3}-\frac{3 V_{0}}{4 \pi}\right) \cos \left(\frac{\pi t}{T_{b}}\right)+\frac{T_{b}}{\pi}\left(a_{m}^{3}-\frac{3 V_{0}}{4 \pi}\right)\right]
$$

For fluid at the same depth with the bubble center, where $\cos \alpha$ equals to 0 , Eq. (27) can be reduced to

$$
u_{r}=\frac{1}{3 S^{2}} \frac{\pi}{T_{b}}\left(a_{m}^{3}-\frac{3 V_{0}}{4 \pi}\right) \cos \left(\frac{\pi t}{T_{b}}\right) .
$$




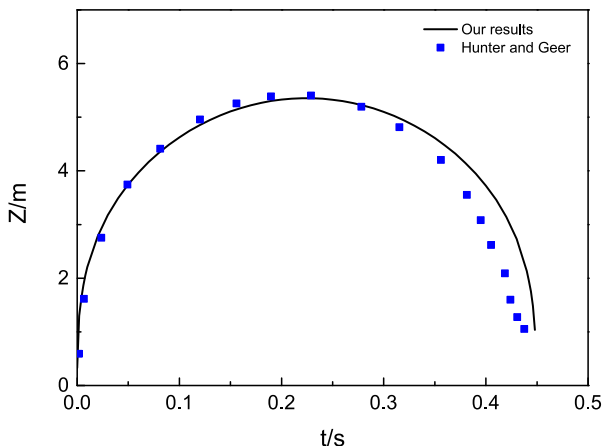

(a) Radius history of the bubble

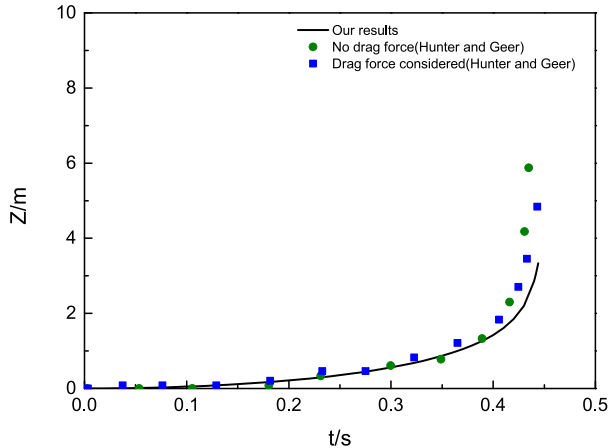

(b) Migrating displacement history

Fig. 3. Bubble dynamics in its first period for $226.7 \mathrm{~kg}$ TNT detonated at $45.7 \mathrm{~m}$ below the sea surface. The solid curves in (a) and (b) are the history of the bubble radius obtained by Eq. (20) and the migrating displacement history of the bubble center calculated by Eq. (25), respectively. The dots are the corresponding data from Hunter and Geers [11], which considered the compressibility in both the external water and the internal gas. Good agreements are achieved in the comparison.

At last, by dropping $3 V_{0} /(4 \pi)$ as it is sufficient small compared with $a_{m}^{3}$, the maximal speed $u_{m}$ at the same depth with the bubble center can be estimated by

$$
u_{m}=\frac{a_{m}^{3}}{3 S^{2}} \frac{\pi}{T_{b}} .
$$

As the experimental data are lacking for the radial velocity history, we checked the maximal speed $u_{m}$ at the same depth with the bubble center for two explosion situations. One is $226.7 \mathrm{~kg}$ TNT detonated at $45.7 \mathrm{~m}$ below the sea surface, and the other one is $1 \mathrm{~kg}$ TNT detonated at the depth of $5 \mathrm{~m}$. For the former, $u_{m}$ at $11.25 \mathrm{~m}$ far from the charge center is $3.20 \mathrm{~m} / \mathrm{s}$ by Eq. (29), while it was $3.04 \mathrm{~m} / \mathrm{s}$ given by Hunter and Geers [11]. And the results for the latter at different distances are listed in Table 1 showing acceptable agreement with those given by MSC.Dytran as well. Thus, Eq. (29) of $u_{m}$ can be used for estimating the dimensionless numbers in Eq. (16).

\section{Underwater explosion regimes}

\subsection{Discussion on the scaling law}

Now let's give some discussion on the relation of Eq. (16), which contain four dimensionless numbers: $f_{n}, E_{n}, G_{n}$ and $I_{n}$ at its right side.

$f_{n}$ mainly represents the effect of bubble pulsation frequency. As we know, the bubble pulsation frequency $f_{b}$ considerably affects the global response when it is close to the structural eigen-frequency and resonance occurs. For a submarine with the length varies from $60 \mathrm{~m}$ to $120 \mathrm{~m}$ and the equivalent thickness from $5 \mathrm{~cm}$ to $25 \mathrm{~cm}$, its lowest eigen-frequency is estimated to range from $25 \mathrm{~Hz}$ to $55 \mathrm{~Hz}$. In contrast, $f_{b}$ is between $5 \mathrm{~Hz}$ and $15 \mathrm{~Hz}$ for charge mass ranging from $100 \mathrm{~kg}$ to $1000 \mathrm{~kg}$ and explosion depth from $100 \mathrm{~m}$ to $400 \mathrm{~m}$. Therefore, no overlapping of the two frequencies ranges exists indicating that no intense resonance occurs. What's more, if the explosion happens at a depth nearby the typical submarine's operating depth (say from $250 \mathrm{~m}$ to $350 \mathrm{~m}$ ), the variation of $f_{b}$ is less than $3 \mathrm{~Hz}$. Accordingly, the effect of $f_{n}$ is insignificant for a typical submarine subjected to the bubble loading near its operating depth. However, it should be noted that for systems in near/intense resonant condition such as a typical frigate-size warship by underwater explosion [1], large amplitude whipping displacements can probably be excited. Consequently, importance of $f_{n}$ should be reconsidered. 
$I_{n}$ in Eq. (12) reflects the effects of rotary inertia, structural geometry and fluid-structure interaction. As we know, comparing with the Euler-Bernoulli beam model, the Timoshenko beam model incorporates two more effects: the shear distortion and the rotary inertia. And the influence of the rotary effect is droppable as it is always less than one third of effect of shear [22].

Finally, $E_{n}$ and $G_{n}$ reflecting the roles of tensile and shear are two new dimensionless numbers we developed in this work. For convenience, $E_{n}$ and $G_{n}$ are named as "tensile number" and "shear number" here, respectively. They include the following four factors: external load, structural geometry, resistance ability to structural deformation and fluid-structure interaction. From Eq. (10), $E_{n}$ is a development of Cauchy number and can be regard as another branch comparing with introducing the geometrical influence into the damage number [17].

Consequently, Eq. (16) is reduced to the scaling law below,

$$
\frac{\sigma_{m}}{\sigma_{Y}}=f\left(E_{n}, G_{n}\right),
$$

in the elastic deformation stage of material. For structure with large slenderness ratio that the shear effect disregarded, Eq. (30) can be further reduced to Eq. (32).

Eq. (30) may also be written in the alternative form

$$
\frac{\sigma_{m}}{\sigma_{Y}}=f\left(E_{n}, \frac{E_{n}}{G_{n}}\right),
$$

which has the advantage that the dimensionless stress will vary only with $E_{n}$ for a given structure. That's because $E_{n} / G_{n}$ equals to $E I /\left(k G A L^{2}\right)$ which is a constant for a given structure. Which also implies that, for structures with similar geometrical shape and size, $E_{n} / G_{n}$ can only affect the result slightly. Thus Eq. (30) or (31) can also be simplified to Eq. (32) for the preceding two situations.

$$
\frac{\sigma_{m}}{\sigma_{Y}}=f\left(E_{n}\right)
$$

can be regarded as the scaling law for predicting the structural elastic global response by pulsating bubble, and $E_{n}$ is the unique response number.

\subsection{Classification of the explosive regimes}

Despite the terms "near-field", "middle-field" and "far-field" are commonly used in the community of engineering, the explosive regimes haven't yet been quantitatively defined so far. To our knowledge, people usually assume

1) The material starts to yield and thus leads to the breakdown of structure immediately for near-field explosion;

2) substantial structure deformation can be detected in the middle-field along with violent structure movement;

3) the deformation of entire structure are found negligible, and only it's overall motion as a rigid-body is concerned with in the far-field.

In some works [11], the near-field and far-field are divided by fifteen times of the charge radius. Evidently, this kind of definition without considering any structure information is not appropriate for the estimation of explosion effects. We propose that the following two aspects should be considered to

Table 1

Maximal radial flow speed predicted by Eq. (29) and MSC.DYTRAN for $1 \mathrm{~kg}$ TNT explode at $5 \mathrm{~m}$ under the water surface.

\begin{tabular}{lllr}
\hline Distance & Eq. (29) & MSC.DYTRAN & Deviation \\
\hline $3 \mathrm{~m}$ & $1.50 \mathrm{~m} / \mathrm{s}$ & $1.51 \mathrm{~m} / \mathrm{s}$ & $0.6 \%$ \\
$4 \mathrm{~m}$ & $0.84 \mathrm{~m} / \mathrm{s}$ & $0.90 \mathrm{~m} / \mathrm{s}$ & $6 \%$ \\
$5 \mathrm{~m}$ & $0.54 \mathrm{~m} / \mathrm{s}$ & $0.60 \mathrm{~m} / \mathrm{s}$ & $10 \%$ \\
\hline
\end{tabular}


demarcate these regimes: One is the structural global response like structural deformation or acceleration response. The other one is the various influencing factors, such as charge mass, explosion distance/depth, structural geometry and material strength. Fortunately, the foregoing scaling law provides us with a relation between the dimensionless stress and $E_{n}$, which incorporates the various influencing factors together. Accordingly, the classification of the three regimes we introduced can be specified as

$$
\left\{\begin{array}{cc}
\text { Near - field : } & \sigma_{m} / \sigma_{Y} \geq 1 \\
\text { Middle - field : } & 0.05<\sigma_{m} / \sigma_{Y}<1, \\
\text { Far - field : } & \sigma_{m} / \sigma_{Y} \leq 0.05
\end{array}\right.
$$

On the basis of the previous discussion, $\sigma_{m} / \sigma_{Y}$ strongly depends on $E_{n}$. Consequently, Eq. (33) can be rewritten in terms of $E_{n}$ as

$$
\left\{\begin{array}{cc}
\text { Near - field : } & \lg E_{n} \leq \lg E_{n Y}, \\
\text { Middle - field : } & \lg E_{n Y}<\lg E_{n}<\lg E_{n R}, \\
\text { Far - field : } & \lg E_{n} \geq \lg E_{n R} .
\end{array}\right.
$$

where, $E_{n Y}$ is the threshold for the near-field corresponding to the yield stress $\sigma_{Y}$, and $E_{n R}$ is the threshold for the far-field corresponding to the stress of $5 \% \sigma_{Y}$. $\lg E_{n}$ can be derived easily for a specific slender structure by substituting Eqs. (17), (18) and (29) into Eq. (10)

$$
\lg E_{n}=\lg \left(\frac{9 K_{2}^{2}}{\pi^{2} K_{1}^{6}}\right)+\lg \left(\frac{I R^{2}}{\left(\rho A+m_{a}\right) L^{4}}\right)+\lg E-\frac{4}{3} \lg C+4 \lg S+\frac{1}{3} \lg (H+10.3) .
$$

On the right hand side of Eq. (35), the first term is a constant, and the other five terms represent the influence of the geometry and the fluid-structure interaction, structural material, TNT mass, explosion distance and explosion depth, severally. The structural material affects $\lg E_{n}$ not only by the elastic modulus as shown in Eq. (35) but also changes the threshold demarcating the regimes via yield stress as noted earlier. Hence the regimes criterion is capable of considering the numerous physical quantities.

Consequently, one can know which regime the explosion belongs to conveniently via Eqs. (34) and (35). On the basis of this criterion, the preliminary analysis of structural global response can be carried out without any complicated calculation as each regime is associated with different features of structural response.

\section{Case studies and discussion: typical submarines}

Based on typical submarine parameters, three beam models in Table 2 with different equivalent thicknesses are used to observe the corresponding near-, middle- and far-field responses. Five groups of explosion cases are designed as shown in Table 3, in which the explosion depth ranges from $250 \mathrm{~m}$ to $325 \mathrm{~m}$ to maintain the influence of $f_{n}$ at a low level and the explosion distances are all no less than $20 \mathrm{~m}$ to ensure the boundary induced bubble jet will not appear hence the spherical bubble assumption is reasonable.

\subsection{Mathematical solution}

The dimensionless displacement $\mathrm{W}(\mathrm{X}, \mathrm{T})$ of Eq. (15) can be decomposed into a linear combination of a set of modes like

$$
W(X, T)=\sum_{j=1}^{N} \chi_{j}(T) \eta_{j}(X)
$$


where $N$ is the number of modes, $\eta_{1}(X)$ is the rigid-body movement mode, $\eta_{2}(X)$ is the rigid rotation mode, and $\eta_{j}(X)(j \geq 3)$ is the free vibration modes satisfying the zero shear force/bending moment boundary conditions at the free ends. Substituting Eq. (36) into Eq. (15), multiplying both sides of Eq. (15) by $\eta_{i}(X)$, and integrating along the $X$ span, we have

$$
[N] \frac{d^{4}[Z]}{d T^{4}}+[M] \frac{d^{2}[Z]}{d T^{2}}+[K][Z]=[F] .
$$

where,

$$
Z_{i}=\chi_{i}(T),
$$

$$
\begin{aligned}
& N_{i j}=\frac{I_{n}}{G_{n}} \int_{0}^{1} \eta_{j}(X) \eta_{i}(X) d X, \\
& M_{i j}=\int_{0}^{1} \eta_{j}(X) \eta_{i}(X) d X-\left(\frac{E_{n}}{G_{n}}+I_{n}\right) \int_{0}^{1} \frac{d^{2} \eta_{j}(X)}{d X^{2}} \eta_{i}(X) d X, \\
& K_{i j}=E_{n} \int_{0}^{1} \frac{d^{4} \eta_{j}(X)}{d X^{4}} \eta_{i}(X) d X, \\
& F_{i}=\frac{I_{n} f_{n}}{G_{n}} \int_{0}^{1} \frac{\partial^{2} \dot{U}}{\partial T^{2}} \eta_{i}(X) d X-\frac{E_{n} f_{n}}{G_{n}} \int_{0}^{1} \frac{\partial^{2} \dot{U}}{\partial X^{2}} \eta_{i}(X) d X+f_{n} \int_{0}^{1} \dot{U} \eta_{i}(X) d X .
\end{aligned}
$$

As the shock has little influence on the global response, the initial conditions can be specified as

$$
\begin{aligned}
& \left.W\right|_{T=0}=0, \\
& \left.\frac{\partial W}{\partial T}\right|_{T=0}=0 .
\end{aligned}
$$

Using Eqs. (43) and (44) together with $\left.\theta\right|_{T=0}=0$ and $\left.\frac{\partial \theta}{\partial T}\right|_{T=0}=0$, the four initial conditions of [Z] are expressed as

$$
\left.[Z]\right|_{T=0}=0,
$$

\section{Table 2}

Parameters of the three beam models. The beam model one is considered as a standard model, and the other two models have a variation on the equivalent thickness of that.

\begin{tabular}{lllllll}
\hline Beam & $L / \mathrm{m}$ & $R / \mathrm{m}$ & $\delta / \mathrm{cm}$ & $E / \mathrm{GPa}$ & $G / \mathrm{GPa}$ & $\sigma_{Y} / \mathrm{MPa}$ \\
\hline 1 & 100 & 7 & 20 & 205 & 80 & 689 \\
2 & 100 & 7 & 10 & 205 & 80 & 689 \\
3 & 100 & 7 & 25 & 205 & 80 & 689 \\
\hline
\end{tabular}


Table 3

Parameters of four groups of explosion situations. The first three groups of situations based on the beam model one are used to observe the influences of TNT mass, explosion depth and distance, while groups four and five for the structural geometry effect.

\begin{tabular}{lllll}
\hline Group & $C / \mathrm{kg}$ & $S / \mathrm{m}$ & $H / \mathrm{cm}$ & Beam \\
\hline 1 & $300,400,500,600,700$ & 30 & 300 & 1 \\
2 & 500 & 30 & $250,275,300,325$ & 1 \\
3 & 500 & $20,30,40,50,60,70,80,90$ & 300 & 1 \\
4 & 500 & $20,30,40,50,70,90$ & 300 & 2 \\
5 & 500 & $20,30,50,70$ & 300 & 3 \\
\hline
\end{tabular}

$\left.\frac{d[Z]}{d T}\right|_{T=0}=0$

$$
\begin{aligned}
& {\left.[N] \frac{d^{2}[Z]}{d T^{2}}\right|_{T=0}=f_{n} \int_{0}^{1} \dot{U} \eta_{i}(X) d X,} \\
& {\left.[N] \frac{d^{3}[Z]}{d T^{3}}\right|_{T=0}=f_{n} \int_{0}^{1} \frac{\partial \dot{U}}{\partial T} \eta_{i}(X) d X .}
\end{aligned}
$$

Solving the four-order system Eq. (37) with the initial conditions given in Eqs. (45),(46),(47),(48), we readily have the global response of the submerged floating slender structure.

\subsection{Criterion of the three regime}

We at first try to validate the scaling law of Eq. (32) for a given structure which means $\sigma_{m} / \sigma_{Y}$ should only depend on $E_{n}$. The relation between $\sigma_{m} / \sigma_{Y}$ and $\lg E_{n}$ of groups 1 to 3 are illustrated in Fig. 4, demonstrating that all of the points $\left(\sigma_{m} / \sigma_{Y}, \lg E_{n}\right)$ fall on an exponential curve and thus validating the scaling law for a given submarine.

Further, we also compared the results of groups 3, 4 and 5 as shown in Fig. 5 to check the influence of equivalent thickness. ( $\sigma_{m} / \sigma_{Y}, \lg E_{n}$ ) of all groups fall on an exponential fitting curve basically. That's because the equivalent thickness is constrained within a narrow band and $E_{n} / G_{n}$ can hardly affect the result. In our models, the equivalent thickness ranges from $10 \mathrm{~cm}$ to $25 \mathrm{~cm}$ hence its board enough for covering typical submarine. Therefore, the scaling law of Eq. (32) still works for typical submarines, and the exponential fitting curve in Fig. 4 is effective for the explosive regime evaluation.

For typical submarines (see Table 2), we have $\lg E_{n Y}$ equals to 1.70 and $\lg E_{n R}$ equals to 3.94 to demarcate three regimes according to Fig. 5. Eventually, the near-, middle- and far-field are divided as

$$
\left\{\begin{array}{cc}
\text { Near - field : } & \lg E_{n} \leq 1.70, \\
\text { Middle - field : } & 1.70<\lg E_{n}<3.94 \\
\text { Far - field : } & \lg E_{n} \geq 3.94 .
\end{array}\right.
$$

In order to give a more intuitionistic idea of the regimes, we present the regime diagrams as shown in Fig. 6 for the beam models 1 and 2. Both near-/middle-field interface and middle-/far-field interface are almost perpendicular to the "Explosion distance-Charge weight" plane, implying that the influence of the explosion depth is insignificant. The projection curves of the interfaces on the "Explosion distance-Charge weight" plane show that the ranges of the near- and middle-fields expand with the increasing charge weight. In other words, more charge at larger explosion distance can produce the 


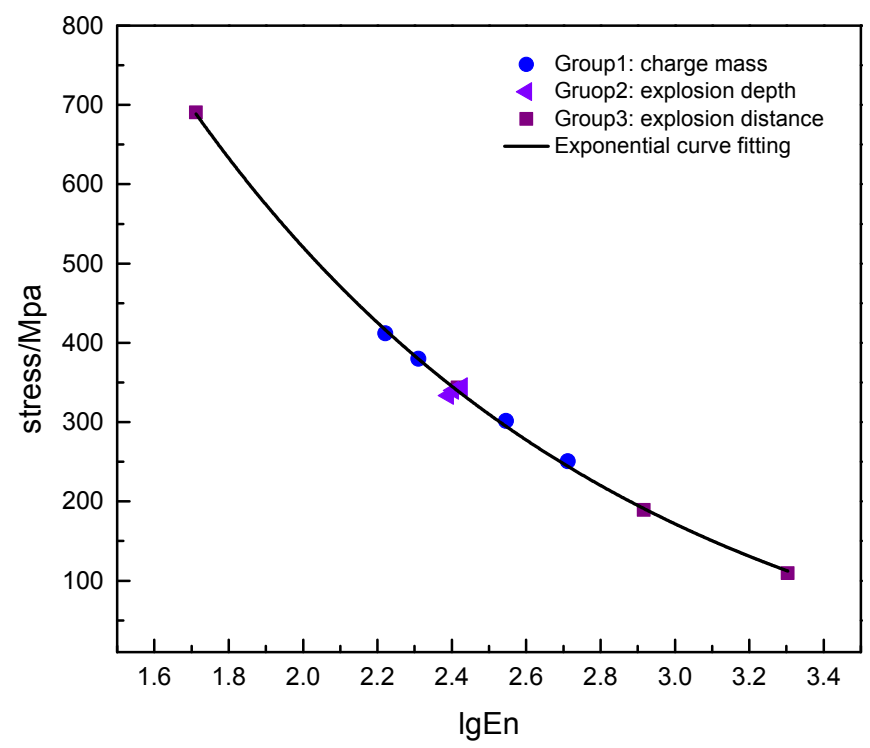

Fig. 4. Relation between $\sigma_{m} / \sigma_{Y}$ and $\lg E_{n}$ for a given structure. The three groups of parameters are associated with different charge mass, explosion distance and explosion depth as given in Table 3. The solid curve is an exponential fitting curve based on the results of the three groups. It shows that $\sigma_{m} / \sigma_{Y}$ strongly depend on the dimensionless number $\lg E_{n}$, thus, the scaling law of Eq. (32) is validated.

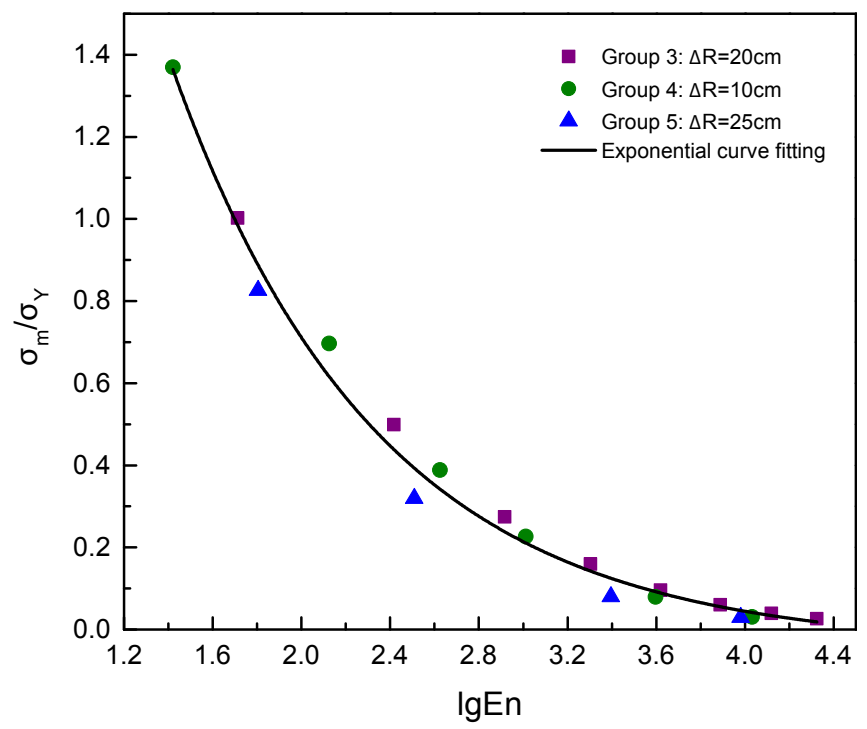

Fig. 5. Relation between $\sigma_{m} / \sigma_{Y}$ and $\lg E_{n}$ for beams with different equivalent thicknesses. The symbols of solid square, circle and triangle indicate the results for groups 3, 4 and 5 as given in Table 3, respectively. The solid curve is an exponential fitting curve based on the results of these three groups. The diagram shows that $E_{n}$ can be regard as the only parameter for the global response of typical submarines by explosion bubble.

same effects as that by less charge at nearer explosion distance. From Eq. (35) we can find out the influence levels of charge weight, explosion distance and explosion depth quantitatively: for the same percent of variation, the influence of charge weight is 4 times larger than that of the explosion depth, but only one third of the influence of explosion distance. 


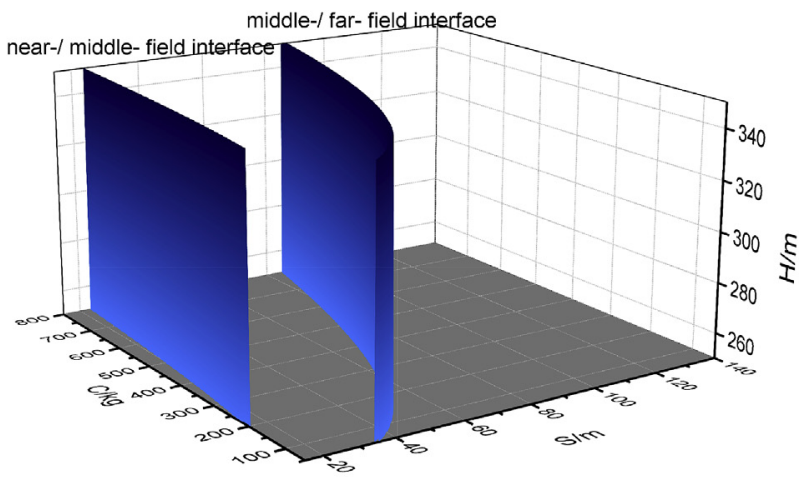

(a)Beam model $1(\delta=20 \mathrm{~cm})$

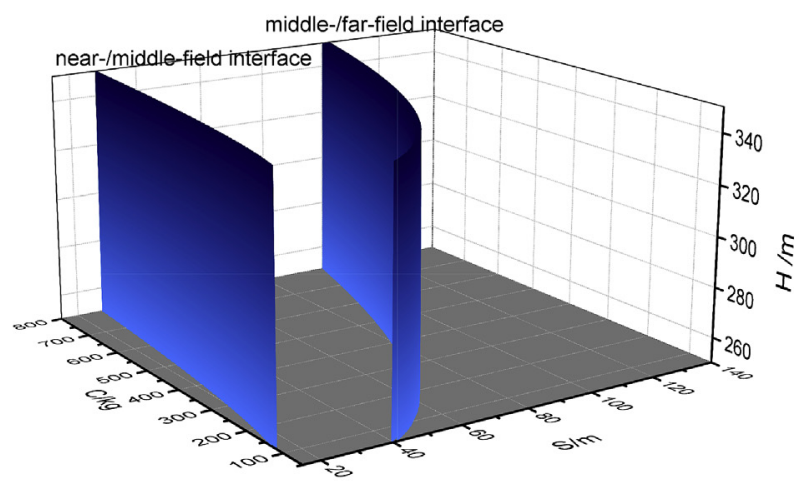

(b) Beam model $2(\delta=10 \mathrm{~cm})$

Fig. 6. The regimes of near-/middle-/far-fields for the beam models 1 and 2 . The three fields are divided according to Eq. (49), and the parameters of the two beam models are shown in Table 2. The three axes are charge weight $C$ in kilogram, explosion distance $S$ in meter and explosion depth $H$ in meter, respectively. Thus, one can easily distinguish which regime the explosion belongs to for any given explosion situation according to those regime diagrams.

The three fields of the beam model 2 in Fig. 6(b) seem to be a "translation outward", as compared to that of the beam model 1 in Fig. 6(a), which means beam model 1 can bear more severe explosive loading than beam model 2 . Take $500 \mathrm{~kg}$ TNT detonated at explosion depth of $250 \mathrm{~m}$ as an example. The critical explosion distance dividing the near-field and the middle-field is $20 \mathrm{~m}$ and $25 \mathrm{~m}$ for beam model 1 and 2. Similarly, the critical explosion distance dividing the middle-field and the far-field is $75 \mathrm{~m}$ and $88 \mathrm{~m}$ for them.

In the same manner, the regime diagram for any specific submerged slender structure can be drawn quantitatively and used for structural global response analysis.

\subsection{Characteristics of the middle- and far-fields}

Here we present the amplitude and distribution of the displacement in Fig. 7, also that of the speed and acceleration in Fig. 8 for different regimes.

The displacements of the middle- and far-fields explosion for parameters group 3 and group 4 in Table 3 are provided in Fig. 7. For the middle-field explosion in Fig. 7(a), the global elastic deformation is violent leading to the non-uniformly distributed displacements. Meanwhile the global elastic response of far-field explosion as shown in Fig. 7(b) is less significant and the entire structure can be regarded as a rigid-body. Both the centerline displacements of middle- and far-fields are toward the 


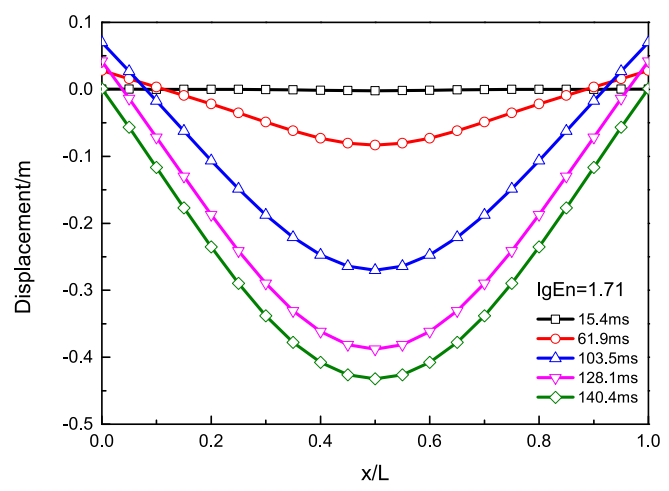

(a) Middle-field explosion

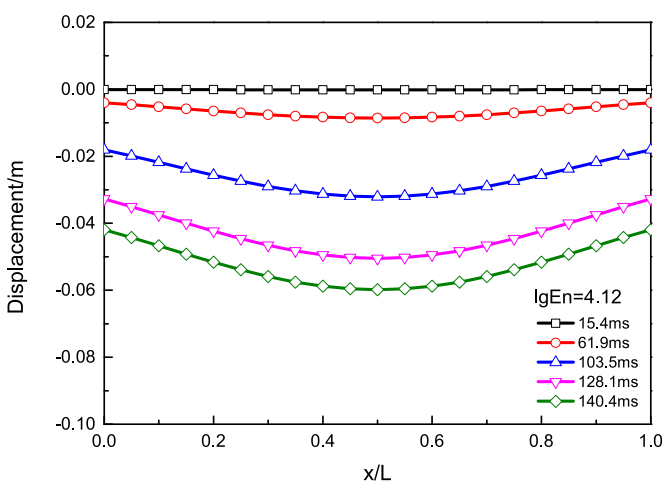

(b) Far-field explosion

Fig. 7. Typical structural centerline displacements of middle- and far-fields at different times. The abscissa represents the dimensionless length of the beam, and the negative ordinate represents the direction of the displacements is the same with the negative Y-axis in Fig. 1. The global elastic response is violent in the middle-field, while rigid motion dominates the structural response of the far-field.

charge, which is caused by the bubble's suction effect. The bubble's suction effect can be explained by Eq. (7) preliminarily which indicates the transverse force of a cross-section depends on both the flow acceleration and its own movement. As we known, in the bubble's expanding phase, the outward flow acceleration reverses when the internal gas pressure becomes less than hydrostatic, and the inward flow acceleration turns back to outward when internal gas pressure exceed hydrostatic in the contracting phase. It is estimated that in over 80 percent of the first cycle, the bubble is over expanding corresponding to the inward flow acceleration. Thus, a long suction effect treads to be produced. Though duration of the suction effect may not be as long as that of the inward acceleration, it is capable of causing inward structural global response like sagging. This sagging liked response may ends at the beginning of the second bubble cycle. But the estimation relies on the modeling accuracy of the bubble's extreme collapse moment strongly, which is beyond this work.

The non-uniformly distributed acceleration/speed peaks for the middle-field explosion are presented in Fig. 8. The maximal acceleration at the free ends can reach as high as $17 \mathrm{~g}$. The maximal speed in the middle section can be up to $5.2 \mathrm{~m} / \mathrm{s}$. Additionally, when the explosion is close to the near-field, for the cases that $\lg E_{n}$ equals to 1.71 and 2.41, directions of the acceleration peaks in the middle part turn to positive as shown in Fig. 8(a) owing to the severe global elastic response.

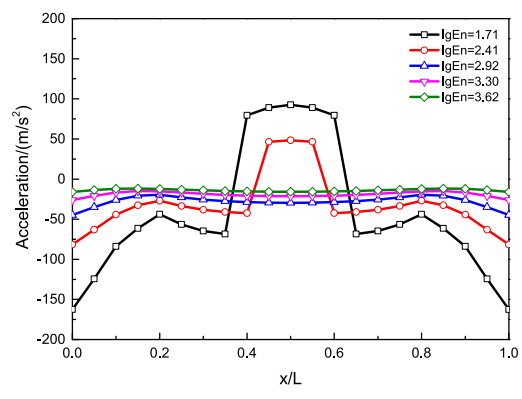

(a) Distribution of acceleration peaks

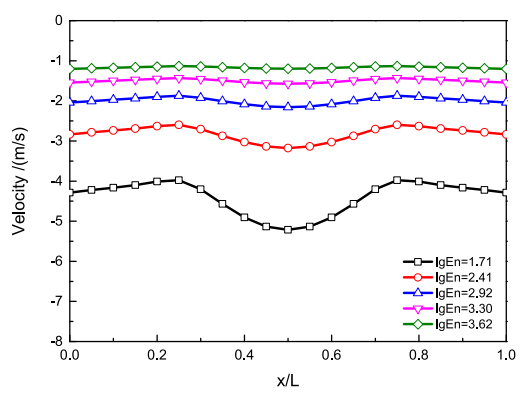

(b) Distribution of speed peaks

Fig. 8. Distribution of acceleration peaks and speed peaks along the structural centerline for the middle-field. Where, peak means the maximal value in the first bubble period. From (a), the global elastic response is significant leading to the large amplitude of the non-uniformly distributed acceleration. While in (b), the distribution of the speed peaks is much gentler than that of the acceleration peaks. Thus, the influence of the global elastic deformation is weaker for the speed response comparing with that for the acceleration response. 


\section{Summary and conclusions}

In this study, we present the regimes of underwater explosion for a submerged slender structure by pulsating bubble. Near-, middle- and far-fields are identified according to structural global responses. In the near-field, the material starts to yield, thus leading to structure breakdown immediately. In the middle-field, violent global elastic response can be found in addition to substantial movement. In contrast, attentions should be paid to overall structural motion as a rigid body in the far-field.

Dimensionless parameters are found out by both similarity analysis and governing equation derivation. Further examination, a dominant parameter $E_{n}$ incorporating the influences of external load, structural geometry, resistance ability to structural deformation and fluid-structure interaction is distinguished to establish a scaling law, which is validated in the case studies. Based on the scaling law, regime criterions of the three fields are further examined.

In case studies, three beam models corresponding to typical submarines are performed. We have provided a quantitative criterion for demarcating these three regimes of near-, middle- and far-fields along with corresponding diagrams. In addition, the structural response features of the middle-/farfields are computed and analyzed. The global response can be regarded as the combination of structural global elastic deformation and rigid-body motion. In the middle-field, both acceleration and speed distribute non-uniformly along the axis direction. And the maximal speed/acceleration can reach as high as $5.2 \mathrm{~m} / \mathrm{s}$ and $17 \mathrm{~g}$.

\section{References}

[1] Vernon TA. Whipping response of ship hulls from underwater explosion bubble loading. Tech. Rep.; DTIC Document. 1986.

[2] Heaton K. The effects of non-sphericity and radiative energy loss on the migration of the gas bubble from underwater explosions. Tech. Rep.; DTIC Document. 1986.

[3] Geers TL, Hunter KS. An integrated wave-effects model for an underwater explosion bubble. J Acoust Soc Am 2002;111(4): 1584-601.

[4] Geers TL, Park CK. Optimization of the g\&h bubble model. Shock Vib 2005;12(1):3-8.

[5] Klaseboer E, Hung K, Wang C, Wang C, Khoo B, Boyce P, et al. Experimental and numerical investigation of the dynamics of an underwater explosion bubble near a resilient/rigid structure. J Fluid Mech 2005;537:387-413.

[6] Zhang A, Yao X, Yu X. The dynamics of three-dimensional underwater explosion bubble. J Sound Vib 2008;311(3): $1196-212$.

[7] Wang Q. Non-spherical bubble dynamics of underwater explosions in a compressible fluid. Phys Fluids (1994-present) 2013;25(7):072104.

[8] Barras G, Souli M, Aquelet N, Couty N. Numerical simulation of underwater explosions using an ALE method. The pulsating bubble phenomena. Ocean Eng 2012;41:53-66.

[9] Liu M, Liu G, Lam K, Zong Z. Smoothed particle hydrodynamics for numerical simulation of underwater explosion. Comput Mech 2003;30(2):106-18.

[10] Hicks A. The theory of explosion induced ship whipping motions. Tech. Rep.; DTIC Document. 1972.

[11] Hunter KS, Geers TL. Pressure and velocity fields produced by an underwater explosion. J Acoust Soc Am 2004;115(4): $1483-96$.

[12] Zhang N, Zong Z. The effect of rigid-body motions on the whipping response of a ship hull subjected to an underwater bubble. J Fluids Struct 2011;27(8):1326-36.

[13] Zhang N, Zong Z. Hydro-elastic-plastic dynamic response of a ship hull girder subjected to an underwater bubble. Mar Struct 2012;29(1):177-97.

[14] Zhang N, Zong Z, Zhang W. Dynamic response of a surface ship structure subjected to an underwater explosion bubble. Mar Struct 2014;35:26-44.

[15] Zhang Zh, Wang Y, Zhang Lj, Yuan Jh, Zhao Hf. Similarity research of anomalous dynamic response of ship girder subjected to near field underwater explosion. Appl Math Mech 2011;32:1491-504.

[16] Brett JM, Yiannakopolous G. A study of explosive effects in close proximity to a submerged cylinder. Int J Impact Eng 2008; 35(4):206-25.

[17] Zhao YP. Suggestion of a new dimensionless number for dynamic plastic response of beams and plates. Arch Appl Mech $1998 ; 68(7-8): 524-38$.

[18] Han SM, Benaroya H, Wei T. Dynamics of transversely vibrating beams using four engineering theories. J Sound Vib 1999; 225(5):935-88.

[19] Joubert P. Some aspects of submarine design. Part 1. Hydrodynamics. Tech. Rep.; DTIC Document. 2004.

[20] Zong Z. Dynamic plastic response of a submerged free-free beam to an underwater gas bubble. Acta Mech 2003;161(3-4): 179-94.

[21] Cole RH. Underwater explosions. New York: Dover Publications; 1965.

[22] Riley M. Analytical solutions for predicting underwater explosion gas bubble behaviour. Tech. Rep.; DTIC Document. 2010. 